\title{
Éditorial : Les trois temporalités de l'anglais de spécialité
}

Anthony Saber

\section{(2) OpenEdition}

1 Journals

Édition électronique

URL : http://journals.openedition.org/asp/5019

DOI : 10.4000/asp.5019

ISSN : 2108-6354

Éditeur

Groupe d'étude et de recherche en anglais de spécialité

\section{Édition imprimée}

Date de publication : 1 novembre 2017

Pagination : 1-4

ISSN : 1246-8185

\section{Référence électronique}

Anthony Saber, «Éditorial : Les trois temporalités de l'anglais de spécialité », ASp [En ligne], 72 | 2017, mis en ligne le 01 novembre 2017, consulté le 02 novembre 2020. URL : http:// journals.openedition.org/asp/5019; DOI : https://doi.org/10.4000/asp.5019

Ce document a été généré automatiquement le 2 novembre 2020.

Tous droits réservés 


\title{
Éditorial : Les trois temporalités de l'anglais de spécialité
}

\author{
Anthony Saber
}

1 Les articles que vous pourrez consulter dans le présent numéro d'ASp, dont plusieurs sont issus du colloque du GERAS organisé en mars 2017 à l'Université de Lyon ${ }^{1}$, illustrent bien à notre sens les trois temporalités de l'anglais de spécialité, toutes trois nécessaires et complémentaires.

2 La première temporalité est celle de l'ancrage théorique : on le sait, l'anglais de spécialité cherche fréquemment des cadres d'analyse et de réflexion dans d'autres disciplines, dont la linguistique systémique fonctionnelle. À cet égard, les notes de lecture proposées par Tiffany Jandrain ${ }^{2}$ sur deux ouvrages de référence, Halliday's Introduction to Functional Grammar de Michael A.K. Halliday et Christian M.I.M. Matthiessen (2004), et Register, Genre, and Style de Douglas Biber et Susan Conrad (2009), confrontent la notion de "registre " à d'autres notions proches ou apparentées: normes, usages, genre et style par exemple. Le travail d'ancrage théorique est aussi une œuvre de stabilisation des notions, dont la portée et l'utilité seront démultipliées si elles font l'objet d'un consensus croissant, ce qui exige - c'est là l'entreprise de T. Jandrain - un inventaire préalable des différentes acceptions existantes. L'ancrage théorique préside aussi au travail mené par Andreas Pichler sur les termes juridiques liés aux contrats de conveyancing (une procédure de transfert de propriété) en anglais : A. Pichler nous propose une réflexion sur un possible "gradient de monosémie » des termes juridiques, dont l'acception se stabilise à mesure que leur périmètre d'emploi se réduit; sa démarche se nourrit d'un utile réexamen de certains concepts fondamentaux de la terminologie : la monosémie au premier chef, mais aussi la morphologie des termes, l'uninotionnalité, la notion de référence et le principe de biunivocité.

La deuxième temporalité peut être définie comme celle de la caractérisation : c'est elle qui est à l'œuvre chez Shaeda Isani comme chez Pascaline Faure dans leurs contributions. Le chercheur en anglais de spécialité s'assigne ici pour tâche de répertorier les caractéristiques distinctives des productions lexicales et discursives, qui sont les manifestations concrètes et observables de la spécialisation de la langue. 
Shaeda Isani s'intéresse au genre des "notes de dégustation » œnologiques, montrant qu'elles recèlent des métaphores synesthésiques particulièrement riches; ce sont en effet de multiples domaines sensoriels qui sont simultanément convoqués par les dégustateurs de vin; couleurs, aspect visuel (robe), caractère, senteurs et saveurs; l'inventivité sans limite des auteurs, souvent sous-tendue par une visée promotionnelle, se déploie au détriment d'une stabilisation des descripteurs œnologiques, peut-être nécessaire afin de tenir un «langage de vérité » auprès du consommateur. Pascaline Faure retrace quant à elle une intention normative (en l'occurrence celle de l'Organisation mondiale de la santé) dans son étude sur la dénomination des nouvelles maladies infectieuses. Traditionnellement, ces dénominations procédaient de multiples matrices lexicogénétiques, telles que l'éponymie (Down's syndrome), l'orthonymie (qui procède de la volonté de décrire les principaux caractères de la maladie, comme dans AIDS, ou acquired immunodeficiency syndrome), et la toponymie (West Nile virus). En 2015, l'OMS publie un document de référence qui invite les découvreurs de nouvelles maladies à adopter une approche exclusivement orthonymique, et définit un certain nombre de normes quant aux caractères que le nom de la maladie devrait contenir ; P. Faure montre cependant que ces préconisations ne sont que peu prises en compte pour le moment.

4 La troisième temporalité de l'anglais de spécialité est, nous semble-t-il, celle de la praxis, qui vise applications et résultats pratiques. La praxis peut par exemple consister à fabriquer des programmes de formation en secteur LANSAD, profilés en fonction d'une analyse des besoins préalable. Elle passe aussi par l'évaluation et le déploiement d'outils numériques de plus en plus puissants dans le champ de l'enseignement LANSAD (tâches pédagogiques ancrées sur des moyens numériques tels que les blogs ou les corpus, dispositifs hybrides ou « distanciels » de formation par le biais de plates-formes en ligne). Cette démarche de mise en œuvre est reflétée dans ce numéro d'ASp par l'article de Marie-Josée Goulet, Michel Simard, Carla Parra Escartìn \& Sharon O'Brien, et par la contribution de François Maniez, qui s'intéressent tous deux aux avancées des moyens de traduction automatisée (TA) au service de l'anglais de spécialité. L'équipe de M.-J. Goulet décrit les apports de ces moyens dans le champ de la rédaction scientifique anglaise sous l'angle de la qualité textuelle. Celle-ci peut être définie, dans le transfert qu'occasionne toute traduction, par certains paramètres tels que la morphologie, la sémantique, le style, la syntaxe, la typographie et la ponctuation. En évaluant les modifications apportées par une réviseuse professionnelle à des résumés d'articles scientifiques, tous rédigés pour une partie dans la langue maternelle des auteurs, et pour une autre partie en anglais - la section en langue maternelle ayant fait l'objet d'une traduction par Google Translate, puis d'une révision préalable par les auteurs eux-mêmes - l'équipe de M.-J. Goulet ne constate pas d'augmentation significative du taux de modifications apportées dans la partie traduite automatiquement, même si les interventions de la réviseuse ne sont pas exactement de même nature que dans la partie directement rédigée en langue anglaise. Ils concluent donc à la pertinence de la traduction automatique comme aide à la rédaction scientifique en anglais, d'autant que la très prometteuse technologie des réseaux neuronaux (Turovski 2016) vient de supplanter les algorithmes traditionnels de Google Translate. François Maniez se penche quant à lui sur l'efficacité des outils de traduction automatique ou d'alignement de corpus (notamment Linguee) pour transférer en français des participes passés prémodifiés par un nom (evidence-based, drug-induced, receptor-mediated), fréquents dans la langue médicale anglaise contemporaine. Les outils 
de TA peinent traditionnellement à traiter ces items au sémantisme compact, qui nécessitent souvent d'être "dépliés" par un étoffement en français. Les résultats obtenus par les moyens de TA semblent très encourageants en la matière, même si le jugement d'un traducteur expérimenté reste souvent nécessaire pour évaluer et valider les solutions obtenues.

Ce numéro, que complètent quatre recensions de John Humbley, Andreas Pichler, Marion Bendinelli et Pascaline Dury, reflète donc bien à notre sens la temporalité trinitaire de l'anglais de spécialité en tant que discipline de la connaissance: sa temporalité est faite de ces moments successifs, qui sont autant d'étapes dans la construction de doctrines, de savoirs et de pratiques; mais n'oublions pas non plus le sens premier de ce mot, c'est-à-dire ce sur quoi nous avons juridiction ${ }^{3}$, le domaine sur lequel nous exerçons notre autorité ; c'est dans ce double sens, à la fois chronologique et juridictionnel, que la temporalité trouve toute son utilité pour tracer le périmètre de notre discipline.

La rédaction remercie chaleureusement les chercheurs qui ont accepté de relire les articles des numéros 71 et 72. Grâce à leurs commentaires, ils ont apporté une aide précieuse aux auteurs et à l'équipe éditoriale. Notre reconnaissance s'adresse à : David Banks, Frank Barbin, Ana Bocanegra-Valle, Geneviève Bordet, Alex Boulton, Nigel Bruce, Angela Chambers, Jean-Pierre Charpy, Marion Charret-Del Bove, Pascaline Dury, Laurent Gauthier, Christopher Gledhill, Daniel Henkel, John Humbley, Shaeda Isani, Elisabeth Lavault, Rudy Loock, Alexandre Mallard, Alexandra Mestivier-Volanschi, Philippe Millot, Carla Parra, Jacqueline Percebois, Michel Petit, Catherine Resche, Isabelle Richard, Elisabeth Rowley-Jolivet, Gail Taillefer, Simon Taylor, Michel Van der Yeught, Séverine Wozniak.

\section{BIBLIOGRAPHIE}

BIBER, Douglas \& Susan CONRAD. 2009. Register, Genre, and Style. Cambridge/New York : Cambridge University Press.

HALLIDAY, Michael A.K. \& Christian M.I.M. MATTHIESSEN. 2004. Halliday's Introduction to Functional Grammar (Fourth Edition). Oxon/New York : Routledge.

TUROVSKY, Barak. 2016. « Found in translation: More accurate, fluent sentences in Google Translate ». Consulté le 2/10/2017 <https://www.blog.google/products/translate/foundtranslation-more-accurate-fluent-sentences-google-translate/>.

WORLD HEALTH ORGANIZATION. 2015. «Best practices for the naming of new human infectious diseases ». Consulté le 2/10/2017 <http://apps.who.int/iris/bitstream/10665/163636/1/WHO _HSE_FOS_15.1_eng.pdf>.

\section{NOTES}

1. « Normes, usages et transgressions en anglais de spécialité ». 
2. Le lecteur trouvera l'ordre de présentation des articles dans le sommaire de ce numéro.

3. On trouve par exemple ce sens chez Bossuet, cité dans le Littré : «Ce qui donnait prétexte aux protestants de préférer leur fidélité à celle des catholiques, c'était la prétention des papes sur la temporalité des rois,» (Bossuet, Déf. var. 1er disc. 55, cité sur <https://www.littre.org/ definition/ temporalité>).

\section{AUTEUR}

\section{ANTHONY SABER}

Rédacteur en chef. anthony.saber@ens-cachan.fr 